\section{A place in the Sun}

\section{G. F. J. Garlick}

Amorphous Silicon Solar Cells. By K. Takahashi and M. Konagai. Translated by F. R. D. Apps. North Oxford Academic*: 1986. Pp.225. £40, $\$ 66$.

THE ElFCIRONICS revolution of the past four decades began with the invention of the transistor, and has rested squarely on the ability to produce large, flawless single crystals of semiconductors, notably silicon. The photovoltaic cell, which can convert some of the sunlight striking it into energy, is a product of the same age. Also made from large single crystal silicon, it was developed for use in space as a power source for satellites, and there is no doubt that it works. Solar cells have reached efficiencies of 15 per cent under space solar flux, and silicon cells with an efficiency of 18 per cent or more have been developed in the laboratory. However, although silicon is an abundant chemical element, silicon solar cells are expensive for general terrestrial use. This led to an extensive search for solar cells based on other semiconducting materials, including microcrystalline structures and, in the 1970s, amorphous silicon. When Carlson produced an amorphous silicon cell with an efficiency of 5.5 per cent at the RC.A Laboratories in Princeton (reported in 1977), this instigated a spread of interest throughout the world.

Amorphous Silicon Solar Cells, translated from Japanese, gives an insight into progress to date, especially in Japan. Half of the chapters discuss solar power, solar cells of the single crystal variety, problems of production and cost reduction, and power production systems. This treatment gives a good background and perspective for the last three chapters which concentrate on amorphous silicon cells; but it also results in a lack of space for indepth information on cell fabrication, and on research and development leading to higher efficiencies and ease of production.

At first sight, an amorphous semiconductor with its plethora of localized states at the electronic band edges, low carrier mobilities and many trapping states would seem to be a poor candidate for an efficient solar cell. However, as the authors show in an historical review, atomic hydrogen produced in glow discharge deposition takes up incomplete silicon bonds which reduces the intergrain conductivity and makes a $\mathrm{p}-\mathrm{i}-\mathrm{n}$ structure possible. Other techniques are also effective, such as boron inclusion. The latter represents the best cell production to date, especially

*Now a division of Kogan Page, 120 Pentonville Road, I ondon N1 9JN, UK. when a heterojunction to a silicon carbide "window" is included in the front of the cell, which gives more efficient transmission of light into the cell and inhibits carrier loss at the front. This technique, and the glow discharge method, came from Carlson and his co-workers.

There are features of the amorphous silicon cell that limit its performance under solar flux conditions, especially in space. The dominant one, which is not yet understood, is a photoelectronic degradation at these flux levels. However, such problems are not insurmountable. Another amorphous solid, selenium, had an instability problem - it reverts to a crystalline form with time. But effective research removed the problem and selenium went on to make a world impact in the form of a photocopying device (Xerox). The intensive efforts of the Japanese, ably described in this text, will no doubt provide a solution for the amorphous silicon problem - or the reason for

no solution - within the next few years. What the authors of this book do bring out clearly is that large area cells can be produced at cost levels well below those for single crystal cells. The achievement of efficiencies of 10 per cent or more for windowed cells at RCA puts such cells into direct competition with single crystal cells for terrestrial applications. Indeed, we already enjoy battery-less calculators using these cells.

This book, with its elegant colour plates of various cell types, power installations and other appliances, makes a very readable introduction to the amorphous silicon cell. The lack of detailed discussion is offset by references at the end of each chapter which give readers access to other sources of literature on the fascinating development of solid state devices.

G. F. J. Garlick, 267 South Beloit Avenue, Los Angeles, California 90049, USA, is a consultant on the design of new-generation silicon and gallium arsenide solar cells for use in space.

\section{Gauging problems}

\section{Paul Langacker}

Group Structure of Gauge Theories. By L. O'Raifeartaigh. Cambridge University Press: 1986. Pp. 172. £20, \$34.50.

THERE have been remarkable advances in elementary particle physics in the past 15 years. We now have a highly successful and mathematically consistent theory, known as the standard model, which incorporates or predicts all known facts concerning the strong, weak and electromagnetic interactions. Despite its successes, however, the standard model cannot be considered seriously as a candidate for the ultimate theory of Nature, mainly because it contains a large number of arbitrary assumptions and free parameters. There is therefore a great deal of current activity in developing still more fundamental theories, such as grand unified theories, supergravity, Kaluza-Klein theories and superstrings. One difficulty for students and experienced researchers alike is that the standard model and, even more so, these generalizations involve the use of increasingly sophisticated mathematics, especially group theory and topology.

This excellent little book provides one big chunk of the necessary background, namely group theory and its application to gauge field theories. The treatment lies somewhere between that of a review article and that of a textbook, and can best be characterized as a rapid tour through a broad class of topics in group theory, particle physics and gauge field theory.

The first half of the book deals with group theory. All of the standard topics are concisely covered, including elemen- tary definitions, Lie groups and algebras, and representation theory. There is also a brief introduction to the application of global symmetries to particle physics and field theory.

The second half covers the algebraic and group theoretical aspects of gauge theories and spontaneous symmetry breaking. Several chapters are devoted to conventional topics such as the structure of gauge theories, the Higgs mechanism, anomalies, the standard model and grand unification. The most unusual parts of the book are the two final chapters, which employ all of the machinery developed earlier. These are respectively devoted to orbit theory, which is the study of the symmetry-breaking patterns induced by specific Higgs representations, and to the minimization of the Higgs potential. These topics represent difficult and underdeveloped areas of research that have previously not been adequately summarized.

This book is an excellent synopsis of the application of group theory to particle physics. It is crammed with useful information, some of which is hard to find elsewhere. There is a very good reference section and a glossary, and there are exercises for each chapter. The book is generally well written, though it is not without its share of typographical errors and an occasional undefined term, and it should prove very useful to advanced theory students and researchers both as a textbook and a reference work. Less experienced readers should be warned, however, that this is a very concise and dense account. It is not intended as an introduction to field theory and in practice also requires some previous knowledge of group theory.

Paul Langacker is a Professor in the Department of Physics, University of Pennsylvania, Philadelphia, Pennsylvania 19174, USA. 